\title{
Determination of the Cerium Oxidation State Incorporated into MCM-41 Silica Matrix by HR-STEM Imaging and EELS Spectra
}

\author{
Raul Borja-Urby and Oscar A. Gonzalez-Vargas \\ Instituto Politecnico Nacional, Mexico City, Distrito Federal, Mexico
}

Given the current trend for the synthesis of novel 2-dimensional materials to technological and scientific applications, nowadays there is a growing interest to determine the structural and chemical properties of the species a nanometric scale. In the present research we concentrate on the determination of cerium oxidation state isomorphically incorporated into high ordered MCM-41 mesoporous silica to determine its multi-point structural variation. A series of novel Ce-doped MCM-41 catalysts was prepared by varying the Ce loading from $2.9 \mathrm{wt} \%$ to $13.2 \mathrm{wt} \%$ via hydrothermal method using CTAB as surfactant. The hybrid composites were characterized by high angle annular dark field (HAADF) imaging and spatially resolved electron energy-loss spectroscopy (EELS) employing a scanning transmission electron microscope (STEM). The M4,5 energy edges for the Ce in the form of spectrum images (SI) were obtained. First, an elemental characterization of the nonstoichiometric ceria-silicas was done from a clear observation of Ce $M_{4,5}$ peaks $(883,901 \mathrm{eV})$ and the single $\mathrm{Si} K$ peak $(1839 \mathrm{eV})$. The analyzes of the energy-loss near-edge structure (ELNES) of the pair Ce M4,5 peaks provided qualitative information about the $\mathrm{Ce}^{4+} / \mathrm{Ce}^{3+}$ oxidation states of cerium species incorporated into the walls of the mesoporous silica. A previous characterization with the X-ray photoelectron spectroscopy (XPS) technique was done to all oxygendeficient Ce-MCM-41 materials in order to discard electron beam damage in the STEM-EELS analysis. The STEM-EELS spectrum imaging provides a facile method to qualitatively identify the cerium oxidation state grafted in the $\mathrm{Si}-\mathrm{O}-\mathrm{Si}$ framework of mesoporous silica. The analytical results here presented shown that the $\mathrm{Ce}^{3+}$ cation is present in all functional materials as was indicated by the shift of the pair Ce M4,5 peaks to a lower binding energy and the increase of the M5/M4 ratio. The cerium ions were found to change from $\mathrm{Ce}^{4+}$ to $\mathrm{Ce}^{3+}$ owing to oxygen deficiency near to the surface of all nanoparticles. In summary, the Ce M4,5-edge EELS spectra have been used to evaluate the structural variation of cubic $\mathrm{CeO}_{2}$ to an oxygen deficient $\mathrm{Ce}_{2} \mathrm{O}_{3}$ structure incorporated into MCM-41 silica. 
(A)

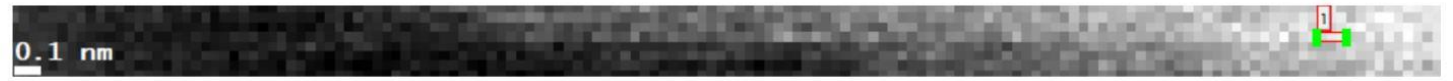

(B)

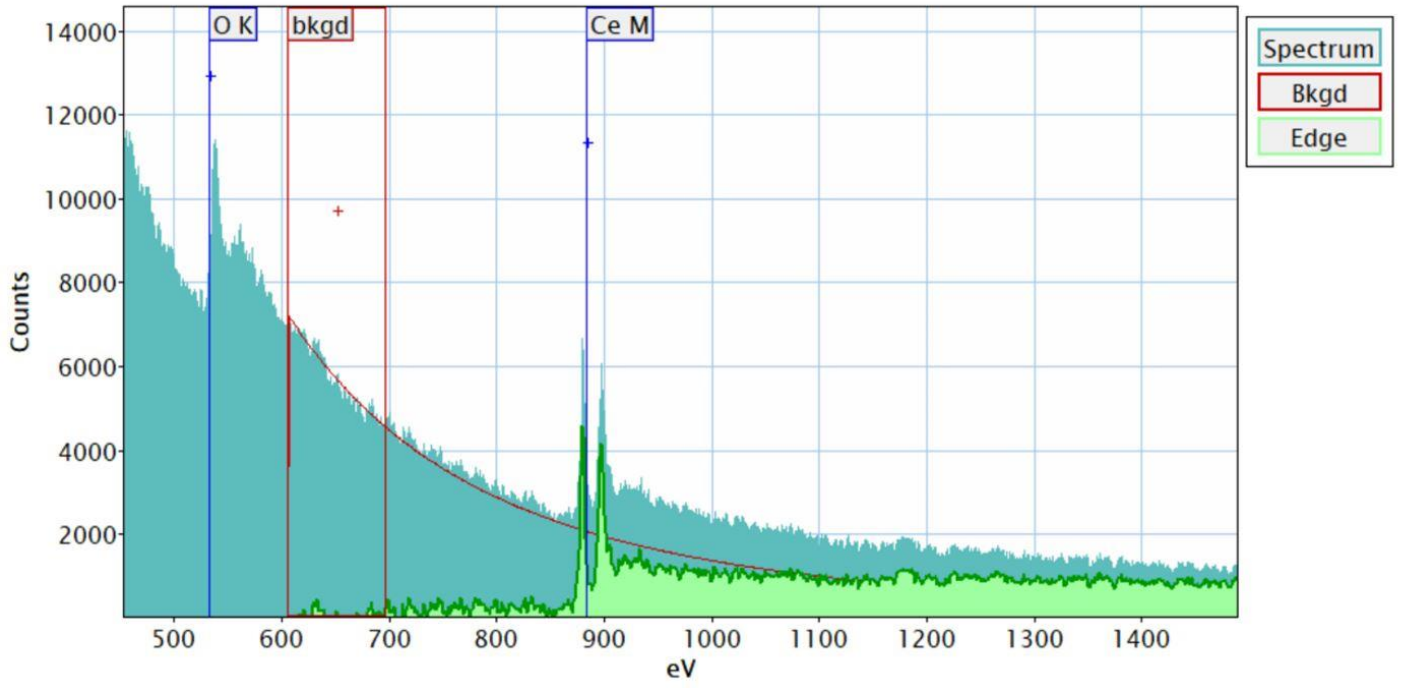

Figure 1. (A) spectrum image (SI), (B) EEL spectrum extracted from SI depicting oxygen K-edge, cerium M4,5 edge, background integration window and cerium signal (green) with background subtraction.

\section{References}

González Vargas, O.A., De los Reyes Heredia, J.A., Suárez-Toriello, V.A. et al. Characterization of structural and optical properties of the mesoporous Ce-MCM-41 hybrid materials. J Mater Sci: Mater Electron 29, 15621-15631 (2018). https://doi.org/10.1007/s10854-018-9154-5

Borja-Urby, R., Paredes-Carrera, S. P., Viltres-Cobas, H., Santiago-Jacinto, P., Paraguay-Delgado, F., Herrera-Pérez, G., ... Morales-Cruz, D. (2019). Confined volume plasmon spatial distribution by low-loss EELS on self-assemble bismuth nanoparticles. Journal of Electron Spectroscopy and Related Phenomena, 237.

https://doi.org/10.1016/j.elspec.2019.146891 